\title{
When is $L(X)$ topologizable as a topological algebra?
}

\author{
by \\ W. ŻELAZKo (Warszawa)
}

\begin{abstract}
Let $X$ be a locally convex space and $L(X)$ be the algebra of all continuous endomorphisms of $X$. It is known (Esterle [2], [3]) that if $L(X)$ is topologizable as a topological algebra, then the space $X$ is subnormed. We show that in the case when $X$ is sequentially complete this condition is also sufficient. In this case we also obtain some other conditions equivalent to the topologizability of $L(X)$. We also exhibit a class of subnormed spaces $X$, called sub-Banach spaces, which are not necessarily sequentially complete, but for which the algebra $L(X)$ is normable. Finally we exhibit an example of a subnormed space $X$ for which the algebra $L(X)$ is not topologizable.
\end{abstract}

We say that a real or complex algebra is topologizable if there is a vector space topology on it such that the product is jointly continuous. In this case there is a basis of neighbourhoods of the origin such that for each such neighbourhood $U$ there is a neighbourhood $V$ with $V^{2} \subset U$, or, more generally, with $V^{n} \subset U$ for any fixed natural $n$. Similarly we say that an algebra is normable if there is a submultiplicative norm on it. Clearly a normable algebra is topologizable. In this paper we are concerned with the question of topologizability of the algebra $L(X)$ of all continuous endomorphisms of a locally convex space $X$. If $X$ is a Banach or normed space, then $L(X)$ is a Banach or normed algebra, which is one of the most important facts in the theory of Banach algebras. However, if $X$ is not a normed space, the algebra $L(X)$ could be topologizable only when $X$ is subnormed, i.e. there is a norm $\|\cdot\|$ on $X$ generating a finer (stronger) topology. Jean Esterle [3] has shown that if the algebra $A(X)$ of all finite-dimensional endomorphisms of $X$ is topologizable, then $X$ is subnormed. Since the topologizability of $L(X)$ implies the topologizability of $A(X)$, the fact that $X$ is subnormed is necessary here. He has also shown in [2] that $X$ is subnormed if and only if $A(X)$ is normable.

In this paper we show that under the assumption of sequential completeness of $X$ the converse is true, so that in this case $L(X)$ is topologizable if and only if $X$ is subnormed and if and only if $L(X)$ is normable. In the proof

2000 Mathematics Subject Classification: 47L10, 46H35. 
we show that for a subnormed $X$ all its continuous endomorphisms are also continuous with respect to the norm $\|\cdot\|$, and then the induced operator norm does the job. Furthermore, we exhibit a class of subnormed spaces $X$ (called sub-Banach spaces), which contains all sequentially complete locally convex spaces and also some spaces which are not sequentially complete and for which $L(X)$ is normable and so topologizable. Here again we show that all operators in $L(X)$ are continuous with respect to $\|\cdot\|$. However we exhibit an example of a subnormed space $X$ for which $L(X)$ is not topologizable.

The topology of any locally convex space $X$ is given by a family of seminorms $\left(|\cdot|_{\alpha}\right)$, thus if $X$ is subnormed with a stronger norm $\|\cdot\|$, then each seminorm $|\cdot|_{\alpha}$ is continuous with respect to $\|\cdot\|$, so that there are positive constants $M_{\alpha}$ such that $M_{\alpha}|x|_{\alpha} \leq\|x\|$ for all $x$ in $X$. Replacing the system $\left(\mid \cdot{ }_{\alpha}\right)$ by $\left(M_{\alpha} \mid \cdot{ }_{\alpha}\right)$ we can assume

$$
\|x\|=\sup _{\alpha}|x|_{\alpha}
$$

for all $x$ in $X$. In this case the closed unit ball $B$ of $\|\cdot\|$ is given by

$$
B=\bigcap_{\alpha} B_{\alpha} \quad \text { where } \quad B_{\alpha}=\left\{x \in X:|x|_{\alpha} \leq 1\right\} .
$$

We call a subnormed space $X$ sub-Banach if its topology can be given by means of a system of seminorms $\left(|\cdot|_{\alpha}\right)$ such that the supremum in (1) is finite for all $x \in X$ and $X$ is complete in the norm $\|\cdot\|$. We shall give an example of a locally convex space which is sub-Banach but not sequentially complete. We can always assume that in an arbitrary subnormed space the norm $\|\cdot\|$ is given by (1) for a certain system of seminorms, but we cannot claim that $X$ is complete in this norm.

We now recall some definitions and facts needed in what follows. Let $X$ be a locally convex space. An absolutely convex bounded subset $B$ of $X$ is called a disc; its Minkowski functional $\|\cdot\|_{B}$ is a norm on the subspace $X_{B}$ spanned by $B$. Such a disc is called a Banach disc if the space $X_{B}$ is complete in $\|\cdot\|_{B}$. For example, if $X$ is sub-Banach, then the unit ball of the norm (1) is a Banach disc. In general, if $B$ is a disc, then the subspace $X_{B} \subset X$ is a subnormed space with the (finer) norm $\|\cdot\|_{B}$ ([6], Proposition 3.2.2). A sequentially complete disc is always a Banach disc ([6], Corollary 3.2.5). A barrel is an absolutely convex, closed and absorbing subset of $X$. A barrelled space is a locally convex space in which every barrel is a neighbourhood of the origin. Every completely metrizable locally convex space is barrelled. Since the set $B$ given by (2) is a barrel and a bounded set (all seminorms $|\cdot|_{\alpha}$ are bounded on it), the Kolmogorov theorem stating that a topological vector space is normed if and only if it is locally convex and locally bounded (has a bounded neighbourhood of the origin) implies that a barrelled subnormed space is normed (this fact is already remarked in [2]). It is known that every barrel absorbs every Banach disc ([6], Proposition 3.2.7). Thus we have 
Theorem A. Let $X$ be a real or complex sequentially complete locally convex space. Then every barrel absorbs every bounded set.

The above follows from the fact that the absolutely convex envelope of a bounded set is bounded, and its closure is also bounded and absolutely convex, so it is a disc. Since a closed subset of a sequentially complete space is sequentially complete, the above disc is a Banach disc and so it is absorbed by every barrel.

Note that if $X$ is subnormed and sequentially complete, then the set $B$ given by (2) is closed, so it is a Banach disc, and $X$ is a sub-Banach space. Thus every sequentially complete subnormed space is sub-Banach.

Call a bounded subset $B$ of $X$ maximal if it absorbs any other bounded set, and call a topological vector space $X$ bornologically normable if there is a norm on it such that the bounded subsets of $X$ coincide with the subsets bounded with respect to this norm.

We can now formulate our first result.

TheOREm 1. Let $X$ be a sequentially complete real or complex locally convex space. Then the following are equivalent.

(i) The algebra $L(X)$ is topologizable.

(ii) The space $X$ is subnormable.

(iii) The space $X$ has a maximal bounded set.

(iv) The algebra $L(X)$ is normable.

(v) The space $X$ is bornologically normable.

Proof. The implication (i) $\Rightarrow$ (ii) is shown in [3], and it holds true without the assumption of sequential completeness. Suppose now that $X$ is subnormable with a norm $\|\cdot\|$, which can be assumed to be of the form (1). The set $B$ given by (2) is closed in the topology of $X$. In fact, if $x_{0}=\lim _{\mu} x_{\mu}$ with $x_{\mu} \in B$, then $\left|x_{\mu}\right|_{\alpha} \leq 1$ for all $\mu$ and $\alpha$, so that $\left|x_{0}\right| \leq 1$ for all $\alpha$ and so $x_{0} \in B$. Consequently, $B$ is a bounded barrel in $X$. By Theorem $\mathrm{A}$ it absorbs all bounded sets in $X$, and (ii) implies (iii).

For (iii) $\Rightarrow$ (iv) it is sufficient to show that every operator $T$ in $L(X)$ is continuous with respect to the norm (1), because then the induced operator norm on $L(X)$ will do the job. Let then $T \in L(X)$. Since the image under a continuous linear map of a bounded set is bounded, it follows that $T(B)$ is bounded and by (iii) there is a positive $C$ such that $T(B) \subset C B$, so that for any $x \in X$ with $\|x\|=1$, we have $\|T x\| \leq C$ and the implication follows. Clearly (iv) implies (i).

To prove (iii) $\Rightarrow(\mathrm{v}$ ) assume that $S$ is a maximal bounded subset in $X$. Since all seminorms giving the topology of $X$ are bounded on $S$, we can renormalize them so that $|x|_{\alpha} \leq 1$ for all $x \in S$ and all $\alpha$. Thus $S$ is contained in a set $B$ of the form (2), and this set is also a maximal bounded 
set. Consequently, all bounded sets in $X$ are bounded with respect to the norm (1), and all sets bounded in the norm (1) are bounded, since they are absorbed by the bounded set (2). Condition (v) follows.

Evidently (v) implies (iii), since every normed space has a maximal bounded set, namely the unit ball of its norm. This completes the proof.

We shall show later that in the absence of sequential completeness condition (ii) implies none of (i), (iii), (iv) and (v).

We now provide the reader with two examples of subnormed spaces which are not normed. It turns out that both are in a natural way multiplicatively convex algebras and they were considered by Michael in [4].

EXAMPLES 2. 1. Let $X$ be the space $C[0,1]$ equipped with the seminorms $|\cdot|_{s}$, where $s$ is a sequence $\left\{\xi_{i}\right\}$ with $\xi_{i} \rightarrow \xi_{0}, 0 \leq \xi_{i} \leq 1$ and $|x|_{s}=$ $\max _{i}\left|x\left(\xi_{i}\right)\right|$. It is a complete non-metrizable locally convex space and it is subnormed with the usual norm in $C[0,1]$.

2 . Denote by $\Omega$ the set of all countable ordinals provided with the segment topology (the basis of open sets consists of the open segments $(\alpha, \beta)$ with $\alpha<\beta)$. Put $X=C(\Omega)$, the vector space of all continuous scalar functions on $\Omega$ with seminorms $|x|_{\alpha}=\max _{0 \leq t \leq \alpha}|x(t)|, \alpha \in \Omega$ (it is well known that all continuous functions on $\Omega$ are bounded). It is a nonmetrizable complete locally convex space which is subnormed with the norm $\|x\|=\max \{|x(t)|: t \in \Omega\}$.

REMARK. It is not hard to see that in the above examples the convergence of sequences in the finer norm (1) is equivalent to the convergence in the original topology. Such an equivalence does not hold true if the space in question is only sequentially complete (e.g. if $X=L(E)$ for a Banach space $E$, provided with the topology of strong convergence of operators, and with the operator norm as the finer norm). Thus we may ask the following

Question. Let $X$ be a complete locally convex space which is a subnormed space with the finer norm of the form (1). Is it true that a sequence of elements of $X$ is convergent in the norm (1) if and only if it is convergent in the original topology of $X$ ?

We now show that $L(X)$ is normable for a class of locally convex spaces $X$ which are not necessarily sequentially complete.

Proposition 3. Let $X$ be a real or complex locally convex space which is sub-Banach. Then the algebra $L(X)$ is normable.

Proof. Let $\|\cdot\|$ be the norm of the form (1) under which $X$ is a subBanach space. As in the proof of Theorem 1, we shall be done if we show that each operator $T \in L(X)$ is continuous with respect to the norm (1). So suppose that some operator is not, and try to get a contradiction. There 
exists a sequence $\left(z_{i}\right) \subset X$ with

$$
\lim \left\|z_{i}\right\|=0
$$

and $\left\|T z_{i}\right\| \geq C>0$. Replacing, if necessary, $z_{i}$ by $\left\|z_{i}\right\|^{-1 / 2} z_{i}$ we can assume

$$
\lim \left\|T z_{i}\right\|=\infty \text {. }
$$

We choose inductively a subsequence $\left(x_{i}\right) \subset\left(z_{i}\right)$ in the following way. Choose first $z_{i_{1}}$ so that $\left\|z_{i_{1}}\right\|<2^{-1}$ and $\left\|T z_{i_{1}}\right\|>1$. By (1) there is an $\alpha_{1}$ such that $\left|T z_{i_{1}}\right|_{\alpha_{1}}>1$. Put $x_{1}=z_{i_{1}}$. Suppose that we have chosen elements $x_{1}, \ldots, x_{n-1}$ and indices $\alpha_{1}, \ldots, \alpha_{n-1}$ in such a way that

(i) $\left\|x_{i}\right\|<2^{-i}$ for $1 \leq i \leq n-1$,

(ii) $\left|T x_{m+i}\right|_{\alpha_{m}}<2^{-i}$ for $1 \leq m \leq n-1$ and $1 \leq i \leq n-m-1$,

(iii) $\left|T\left(\sum_{i=1}^{m} x_{i}\right)\right|_{\alpha_{m}}>m$ for $1 \leq m \leq n-1$.

We now look for $x_{n}$ and $\alpha_{n}$ so that the above relations hold true if we replace $n-1$ by $n$. Relation (3) implies that $\left\|z_{i}\right\|<2^{-n}$ for sufficiently large $i$, say $i \geq i_{1}$. Consider now (ii). The index $n$ occurs here only in the inequalities

$$
\left|T x_{n}\right|_{\alpha_{m}}<2^{m-n} \quad \text { for } 1 \leq m<n .
$$

Relations (1) and (3) imply $z_{j} \rightarrow 0$ in the topology of $X$, and, since $T$ is continuous, we have $\lim \left|T z_{j}\right|_{\alpha_{m}}=0$ for $1 \leq m<n$ and so relations (5) hold true whenever $x_{n}=z_{j}$ and $j$ is sufficiently large, say $j \geq i_{2}$. Consider now (iii). Relation (4) implies $\lim _{j}\left\|T\left(\sum_{i=1}^{n-1} x_{i}\right)+T z_{j}\right\|=\infty$, and so $\left\|T\left(\sum_{i=1}^{n-1} x_{i}\right)+T z_{j}\right\|>n$ for $j \geq i_{3}$. Setting $x_{n}=z_{\max \left(i_{1}, i_{2}, i_{3}\right)}$ we obtain (i) and (ii) with $n-1$ replaced by $n$, and also $\left\|T\left(\sum_{i=1}^{n} x_{i}\right)\right\|>n$. Now (1) implies that there is an $\alpha_{n}$ so that (iii) holds for $m=n$, so that, by the inductive assumption, it holds for $1 \leq m \leq n$, and the inductive construction is complete.

Condition (i) yields $\sum_{i}\left\|x_{i}\right\|<\infty$ and the completeness of $X$ in the norm (1) implies that there is an element $x \in X$ with $\lim _{n}\left(x-\sum_{i=1}^{n} x_{i}\right)=0$. Consequently, $\lim _{n}\left|x-\sum_{i=1}^{n} x_{i}\right|_{\alpha}=0$ for all $\alpha$ and the series converges in $X$. Since $T$ is continuous, we have

$$
T x=\lim _{n} \sum_{i=1}^{n} T x_{i} .
$$

Consequently,

$$
|T x|_{\alpha_{m}}=\lim _{n}\left|\sum_{i=1}^{n} T x_{i}\right|_{\alpha_{m}}
$$

for all $m$. Relations (ii) and (iii) imply that for a fixed $m$ and all $n>m$ we 
have

$$
\left|T\left(\sum_{i=1}^{n} x_{i}\right)\right|_{\alpha_{m}} \geq\left|T\left(\sum_{i=1}^{m} x_{i}\right)\right|_{\alpha_{m}}-\left|T\left(\sum_{i=1}^{n-m} x_{m+i}\right)\right|_{\alpha_{m}}>m-\sum_{i=1}^{n-m} 2^{-i}>m-1 .
$$

Thus $|T x|_{\alpha_{m}} \geq m-1$ for all $m$, so that $\|T x\|=\infty$ by (1). This is the desired contradiction, since $T x \in X$ and $\|\cdot\|$ is a norm on $X$. The conclusion follows.

We now give an example of a space $X$ which is not sequentially complete but satisfies the assumptions of Proposition 3.

EXAMPLE 4 . Let $X$ be the space $C_{\mathrm{b}}(\mathbb{R})$ of all bounded continuous functions on the real line $\mathbb{R}$, provided with the topology given by the seminorms $|x|_{n}=\max _{|t| \leq n}|x(t)|, n=1,2, \ldots$ (the compact-open topology). It is a dense subspace of the space $C(\mathbb{R})$ of all continuous functions on $\mathbb{R}$. It is not sequentially complete, since the spaces considered are metrizable. Clearly $X$ is sub-Banach under the norm (1) equal to $\sup _{t \in \mathbb{R}}|x(t)|$, and so, by Proposition 3, the algebra $L(X)$ is normable. Observe that there is no maximal bounded set in $X$, so that the conclusion of Theorem 1 does not hold true if $X$ is not sequentially complete, since in our case conditions (i), (ii) and (iv) are satisfied while (iii) and (v) are not. Also the completion of $X$ is barrelled and not normed, so it is not subnormed.

The following example gives a locally convex subnormed space $X$ for which the algebra $L(X)$ is not normable (and even not topologizable, as shown in Proposition 6).

Example 5. Denote by $X$ the dense subspace of the complex space $C(\mathbb{R})$ consisting of all compactly supported functions; it is a subspace of the space of the previous example, thus it is also subnormed. We show that the algebra $L(X)$ is not normable. Suppose that it is. Observe first that the operators $T_{\varphi}$ of multiplication by functions $\varphi$ in $C(\mathbb{R})$ are continuous endomorphisms of $X$, so that the algebra $A=C(\mathbb{R})$ can be treated as a subalgebra of $L(X)$. Therefore it is normable with a submultiplicative norm $\|\cdot\|$. However, it is known that $A$ is not normable (cf. [1], Proposition 2.1.14, see also Proposition 2.1.3). Thus $X$ satisfies condition (ii) of Theorem 1, but does not satisfy (iv).

We now show that the algebra $L(X)$ for $X$ as in Example 5 is not topologizable. In the proof we use a modification of the method of [5].

Proposition 6. There exists a locally convex subnormed space $X$ for which the algebra $L(X)$ is not topologizable. More specifically, the space $X$ of all compactly supported continuous functions on $\mathbb{R}$ provided with the compact-open topology has this property. 
Proof. Assume, for contradiction, that there is a (Hausdorff) topology $\tau$ on $L(X)$ making the multiplication jointly continuous. Fix a continuous function $\varphi$ on $\mathbb{R}$ supported in the interval $[1 / 3,2 / 3]$, and denote by $T$ the operator of multiplication by $\varphi$; it is certainly a non-zero element of $L(X)$. Choose a $\tau$-neighbourhood $U$ of the origin in $L(X)$ such that $T \notin U$. Without loss of generality we can assume that $\lambda U \subset U$ for all scalars $\lambda$ satisfying $|\lambda| \leq 1$ (cf. [7], Theorem 1.2.2). By the joint continuity of multiplication we can find a $\tau$-neighbourhood $V$ of the origin so that

$$
V^{3} \subset U \text {. }
$$

Denote by $S$ the left shift on $X$ given by $(S x)(t)=x(t-1), x \in X, t \in \mathbb{R}$. Clearly $S$ is invertible and $S, S^{-1} \in L(X)$. Fix positive $c_{n}$ and $d_{n}$ so that $c_{n} S^{-n} \in V$ and $d_{n} S^{n} T \in V$ for all natural $n$, and put $r_{n}=n /\left(c_{n} d_{n}\right)$. Fix a function $\phi$ in $C(\mathbb{R})$ such that $\phi(t)=r_{n}$ for $t \in[n+1 / 3, n+2 / 3]$ and denote by $Q$ the operator of multiplication by $\phi$. Again we have $Q \in L(X)$. Choose a positive $\varepsilon$ so that $\varepsilon Q \in V$. By (6) we have

$$
c_{n} d_{n} \varepsilon S^{-n} Q S^{n} T \in V^{3} \subset U .
$$

Calculate now the product $S^{-n} Q S^{n} T$. For every $x$ in $X$ we have

$$
\begin{aligned}
\left(S^{-n} Q S^{n} T x\right)(t) & =\left(S^{-n} Q\right)(\varphi(t-n) x(t-n)) \\
& =r_{n} S^{-n}(\varphi(t-n) x(t-n))=r_{n} \varphi(t) x(t)=\left(r_{n} T x\right)(t),
\end{aligned}
$$

since $\varphi(t-n) x(t-n)$ is supported by $[n+1 / 3, n+2 / 3]$ and $\phi$ equals to $r_{n}$ on this interval. Since $x$ was chosen arbitrarily, we obtain $S^{-n} Q S^{n} T=r_{n} T$ for $n=1,2, \ldots$ Now (7) and the definition of $r_{n}$ imply $n \varepsilon T \in U$ for all natural $n$. Since $n \varepsilon>1$ for large $n$, we have $T \in U$, which contradicts our assumption. The conclusion follows.

The above result also shows that in general condition (ii) of Theorem 1 does not imply (i). It also supports the following

Conjecture. Let $X$ be a locally convex subnormed space. Then the algebra $L(X)$ is topologizable if and only if it is normable.

We now show that every subnormed locally convex space is dense in a subnormed space $X$ for which $L(X)$ is normable.

Proposition 7. Let $X$ be a real or complex subnormed space and denote by $\bar{X}$ its completion. Then there is a subnormed space $X_{1}$ with $X \subset X_{1} \subset \bar{X}$ such that the algebra $L\left(X_{1}\right)$ is normable.

Proof. Since $X$ is subnormed, there is a system $\left(|\cdot|_{\alpha}\right)$ of seminorms such that formula (1) gives a norm. The seminorms $|\cdot|_{\alpha}$ extend by continuity to $\bar{X}$ and generate its topology. We denote the extended seminorms with the same symbols. Formula (2) applied to the extended seminorms gives a disc in $\bar{X}$ and it is a Banach disc, since $\bar{X}$ is complete. Let now $X_{1}=X_{B}$ be 
the subspace spanned by $B$. It is a Banach space in the norm (1) and the conclusion follows by Proposition 2.

One of useful results in the theory of Banach spaces states that every Banach space $X$ is a left Banach module over $L(X)$, i.e. the map

$$
(T, x) \mapsto T x
$$

is jointly continuous. Since for some non-normable locally convex spaces $X$ the algebra $L(X)$ is topologizable (it is always topologizable as a semitopological algebra, i.e. with separately continuous multiplication; this can be shown by providing $L(X)$ with the maximal locally convex topology which is given by means of all seminorms, see e.g. [9]), we can ask whether $X$ can be made a topological $L(X)$-module with some topology on $L(X)$, i.e. such that the map (8) is jointly continuous. The negative answer was given in [8], Theorem 10.2. We formulate it as

Proposition 8. Let $X$ be a real or complex locally convex space. If the algebra $L(X)$ can be topologized as a topological vector space in such a way that the map (8) is jointly continuous, then (and only then) $X$ is a normed space.

A. Pirkovskii informed me that he obtained a similar result in his dissertation.

Acknowledgements. I am grateful to Mohamed Oudadess for informing me about the validity of Theorem A. Thanks to him the conjecture I was going to discuss during the ICTAA 3 Conference (2001) in Oulu turned into a positive result. I am also indebted to Graham Allan, Paweł Domański and Lahbib Oubbi for providing me with important references simplifying or replacing some of the original proofs.

\section{References}

[1] H. G. Dales, Banach Algebras and Automatic Continuity, London Math. Soc. Monographs 24, Oxford Univ. Press, 2000.

[2] J. Esterle, Sur la non normabilité de certaines algèbres d'opérateurs, C. R. Acad. Sci. Paris 278 (1974), 1037-1040.

[3] — - Sur la métrisabilité de certaines algèbres d'opérateurs, Rev. Roumaine Math. Pures Appl. 24 (1979), 1157-1164.

[4] E. Michael, Locally multiplicatively-convex topological algebras, Mem. Amer. Math. Soc. 11 (1952).

[5] V. Müller, On topologizable algebras, Studia Math. 99 (1991), 149-153.

[6] P. Pérez Carreras and J. Bonet, Barrelled Locally Convex Spaces, North-Holland, Amsterdam, 1987.

[7] S. Rolewicz, Metric Linear Spaces, PWN, Warszawa, 1984. 
[8] W. Żelazko, Selected Topics in Topological Algebras, Aarhus Univ. Lecture Notes No. 31, 1971.

[9] - On topologization of countably generated algebras, Studia Math. 112 (1994), $83-88$.

Institute of Mathematics

Polish Academy of Sciences

Śniadeckich 8

00-950 Warszawa, Poland

E-mail: zelazko@impan.gov.pl

Received August 1, 2001

Revised version October 3, 2001 